\title{
Hybridizing Energy Conversion and Storage in a Mechanical-to- Electrochemical Process for Self-Charging Power Cell
}

\author{
Xinyu Xue, ${ }^{\dagger} \S$ Sihong Wang, ${ }^{\dagger, \S}$ Wenxi Guo, ${ }^{\dagger}$ Yan Zhang, ${ }^{\dagger}$ and Zhong Lin Wang ${ }^{*}, \dagger$ \\ ${ }^{\dagger}$ School of Materials Science and Engineering, Georgia Institute of Technology, Atlanta, Georgia 30332-0245, United States \\ ${ }^{\ddagger}$ Beijing Institute of Nanoenergy and Nanosystems, Chinese Academy of Sciences, Beijing, China
}

Supporting Information

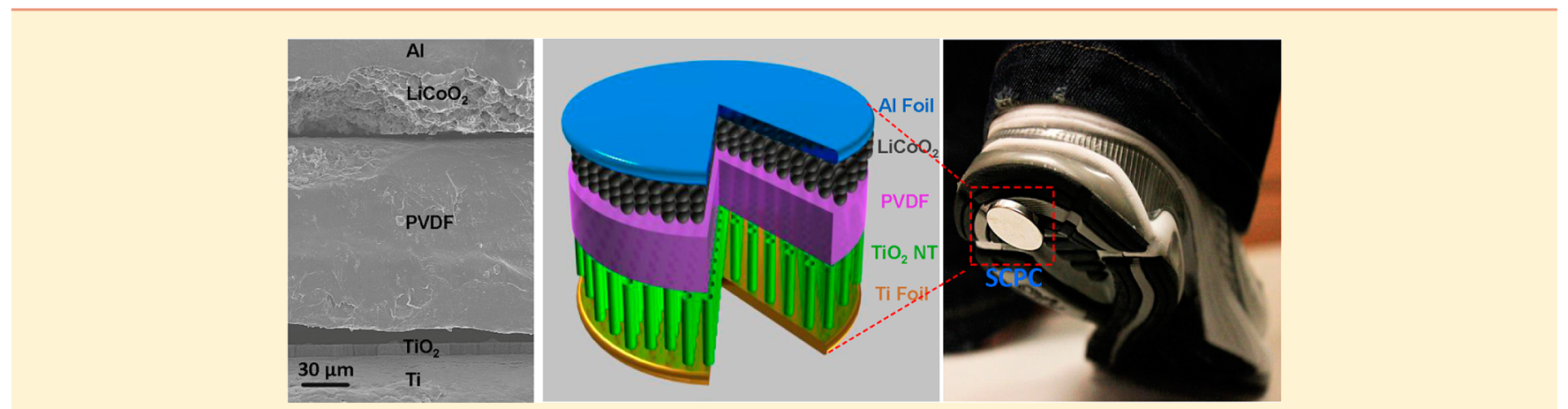

ABSTRACT: Energy generation and energy storage are two distinct processes that are usually accomplished using two separated units designed on the basis of different physical principles, such as piezoelectric nanogenerator and Li-ion battery; the former converts mechanical energy into electricity, and the latter stores electric energy as chemical energy. Here, we introduce a fundamental mechanism that directly hybridizes the two processes into one, in which the mechanical energy is directly converted and simultaneously stored as chemical energy without going through the intermediate step of first converting into electricity. By replacing the polyethylene (PE) separator as for conventional Li battery with a piezoelectric poly(vinylidene fluoride) (PVDF) film, the piezoelectric potential from the PVDF film as created by mechanical straining acts as a charge pump to drive Li ions to migrate from the cathode to the anode accompanying charging reactions at electrodes. This new approach can be applied to fabricating a self-charging power cell (SCPC) for sustainable driving micro/nanosystems and personal electronics.

KEYWORDS: Self-charging power cell, mechanical energy, piezoelectricity, lithium ion battery, electrochemistry

$\mathrm{E}$ nergy conversion and storage ${ }^{1-3}$ are the two most important technologies in today's green and renewable energy science, which are usually separated units designed on the basis of vastly different approaches. As for energy harvesting, depending on the nature of energy sources, such as solar, ${ }^{4-7}$ thermal, ${ }^{8}$ chemical, ${ }^{9}$ and mechanical, ${ }^{10,11}$ various mechanisms have been developed for effectively converting them into electricity. Take smaller scale mechanical energy as an example, piezoelectric nanogenerators (NGs) have been developed into a powerful approach for converting lowfrequency, biomechanical energy into electricity. ${ }^{12-14}$ The mechanism of the NG relies on the piezoelectric potential created by an externally applied strain in the piezoelectric material for driving the flow of electrons in the external load. ${ }^{12}$ As for energy storage, Li-ion battery ${ }^{15-20}$ is one of the most effective approaches, in which the electric energy is stored as chemical energy through the migration of $\mathrm{Li}$ ions under the driving of an externally applied voltage source and the follow up electrochemical reactions occurring at the anode and cathode. ${ }^{21}$

In general, electricity generation and energy storage are two distinct processes that are accomplished through two different and separated physical units achieving the conversions from mechanical energy to electricity and then from electric energy to chemical energy, respectively. Here, we introduce a fundamental mechanism that directly hybridizes the two processes into one, through which the mechanical energy is directly converted and simultaneously stored as chemical energy, so that the nanogenerator and the battery are hybridized as a single unit. Such an integrated self-charging power cell (SCPC), which can be charged up by mechanical deformation and vibration from the environment, provides an innovative approach for developing a new mobile power source for both self-powered systems ${ }^{22}$ and portable and personal electronics.

The experimental design of a self-charging process is based on the characteristics of both piezoelectric and electrochemical properties, as schematically shown in Figure 1a. The device is based on a sealed stainless-steel 2016-coin-type cell, as shown in the highlight of Figure $1 \mathrm{~b}$. The SCPC is composed of three major components: anode, separator, and cathode. The anode is aligned $\mathrm{TiO}_{2}$ nanotube (NT) arrays that are directly grown on a Ti foil. Instead of using the polyethylene (PE) separator ${ }^{23}$

Received: August 2, 2012 


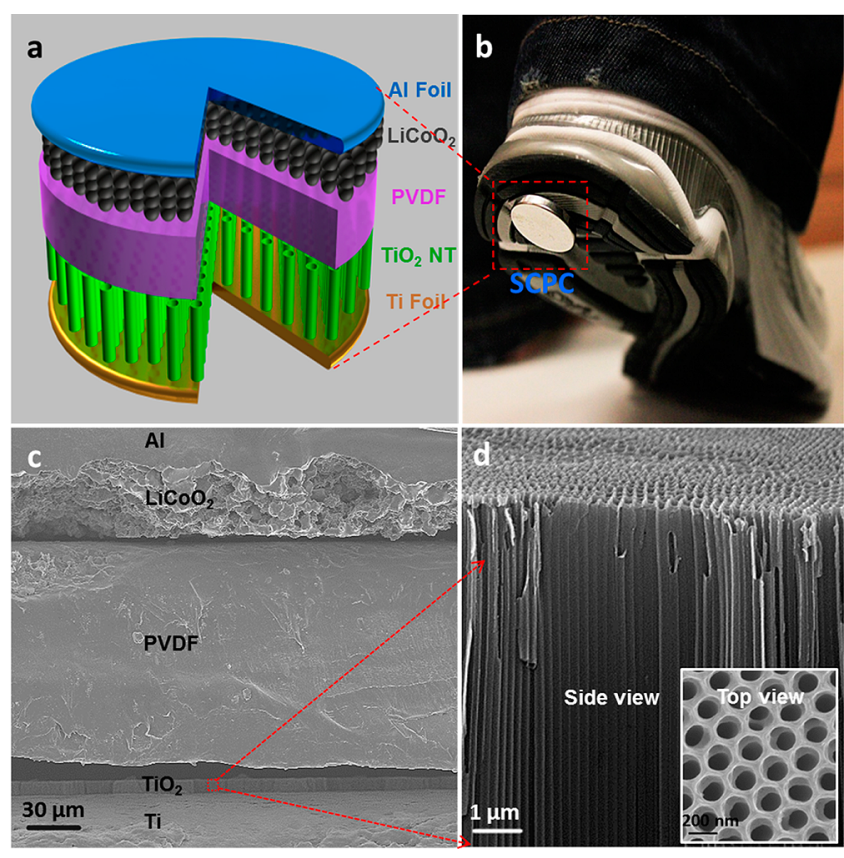

Figure 1. Structure design of a self-charging power cell by hybridizing a piezoelectric nanogenerator and a Li-ion battery. (a) Schematic diagram showing the design and structure of the self-charging power cell. The anode is aligned $\mathrm{TiO}_{2}$ nanotube arrays that are directly grown on Ti foils; a layer of polarized PVDF film performs as the separator; the cathode is a $\mathrm{LiCoO}_{2}$ mixture on aluminum foil. This structure is sealed in stainless-steel 2016-coin-type cells, as shown in the inset. (b) Sticking a power cell on the bottom of a shoe, the compressive energy generated by walking can be converted and stored directly by SCPC. (c) Cross-sectional SEM image of the self-charging power cell, which is composed of aligned nanotubes as anode, piezoelectric polymer film as separator and cathode. (d) Enlarged view of the aligned $\mathrm{TiO}_{2}$ nanotubes. The inset is a top view SEM image of the nanotubes.

as for traditional lithium ion battery, a layer of polarized poly(vinylidene fluoride) (PVDF) film (Measurement Specialties, Inc., U.S.A.) is located above the $\mathrm{TiO}_{2}$ nanotube arrays as the separator. This PVDF film can establish a piezoelectric potential across its thickness under externally applied stress, which not only converts mechanical energy into electricity (Figures S1 and S2, Supporting Information) but also serves as the driving force for the migration of $\mathrm{Li}$ ions. The cathodes are $\mathrm{LiCoO}_{2} /$ conductive carbon/binder mixtures on aluminum foils. Figure $1 \mathrm{c}$ is a cross-sectional scanning electron microscopy (SEM) image of the sandwich structure of the device. The $\mathrm{TiO}_{2}$ nanotube arrays with anatase crystal structure (Figure S3, Supporting Information) were fabricated on Ti substrate using an anodization method with a post-annealing process in air $^{24}$ (Method Summary). The height and diameter of the nanotubes are about $20 \mu \mathrm{m}$ and $100 \mathrm{~nm}$, respectively, as shown in Figure 1d. A commercial piezoelectric PVDF film with a thickness of $\sim 110 \mu \mathrm{m}$ is predominantly composed of $\beta$ phase, which generates the strong piezoelectric effect, and with a small fraction of $\alpha$ phase $^{25}$ (Figure S4, Supporting Information). The PVDF film has been prior poled before assembly into the battery. After placing $\mathrm{LiCoO}_{2}$ cathode with a thickness of 20 $\mu \mathrm{m}$ on the other side, the system was filled with electrolyte ( 1 $\mathrm{M} \mathrm{LiPF}_{6}$ in 1:1 ethylene carbonate:dimethyl carbonate) and finally sealed for measurements. The galvanostatic chargedischarge measurements, with comparison to traditional Li-ion batteries using PE as separators, proved that the power cells act also as a battery system (Figures S5 and S6, Supporting Information). Periodic deformations were applied onto the device in order to charge it up (Figure $1 b$ ), and the voltage and current were monitored simultaneously in both charging and discharging processes.

The working mechanism of the self-charging power cell is an electrochemical process driven by deformation created piezoelectric potential (Figure 2). At the very beginning, the device is at a discharged state, with $\mathrm{LiCoO}_{2}$ as the positive electrode (cathode) material and $\mathrm{TiO}_{2} \mathrm{NTs}$ as the negative electrode (anode), which is the originally fabricated structure of the device, and the $\mathrm{LiPF}_{6}$ electrolyte is evenly distributed across the entire space, as shown in Figure 2a. A PVDF film, which has intimate contacts with both electrodes, serves as the separator and it has the smallest Young's modules among all of the components in the device [PVDF in electrolyte solvent: $\sim 1.2$ $\mathrm{GPa}$ (see section $\mathrm{E}$ in the Supporting Information); $\mathrm{TiO}_{2}: 100$ $\mathrm{GPa}\left(Y_{\mathrm{a}}\right)$ and $266 \mathrm{GPa}\left(Y_{\mathrm{c}}\right) ; \mathrm{LiCoO}_{2}: \sim 70 \mathrm{GPa}$; Ti foil: $100-$ $110 \mathrm{GPa}$; $\mathrm{Al}$ foil: $69 \mathrm{GPa}$; thus, it suffers from the most severe compressive strain when a compressive stress is applied onto the device, as shown in Figure $2 \mathrm{~b}$. We purposely use the PVDF film with the polarity that results in a positive piezoelectric potential (piezopotential) at the cathode $\left(\mathrm{LiCoO}_{2}\right)$ side and negative piezopotential at the anode $\left(\mathrm{TiO}_{2}\right)$ under compressive strain for separating the charges (see section F and Figure S7 in the Supporting Information). Under the driving of the piezoelectric field with a direction from the cathode to the anode, the $\mathrm{Li}$ ions in the electrolyte will migrate along the direction through the ionic conduction paths present in the PVDF film separator for ion conduction in order to screen the piezoelectric field, and finally reach the anode, as shown in Figure 2c (note: a PVDF film is an ionic conductor for $\mathrm{Li}^{+}$, which is why PVDF is used as the base for polymer electrolyte $^{26}$ and also the binder ${ }^{27}$ for electrodes in Li-ion batteries). The decreased concentration of $\mathrm{Li}^{+}$at the cathode will break the chemical equilibrium of the cathode electrode reaction $\left(\mathrm{LiCoO}_{2} \leftrightarrow \mathrm{Li}_{1-x} \mathrm{CoO}_{2}+x \mathrm{Li}^{+}+x \mathrm{e}^{-}\right),{ }^{28}$ so that $\mathrm{Li}^{+}$will deintercalate from $\mathrm{LiCoO}_{2}$, turning it into $\mathrm{Li}_{1-x} \mathrm{CoO}_{2}$ and leaving free electrons at the current collector ( $\mathrm{Al}$ foil) of the cathode electrode. This process is driven by the tendency of establishing new chemical equilibrium (see section $G$ in the Supporting Information). In the meanwhile, under the elevated concentration of $\mathrm{Li}^{+}$at the anode, the reaction at the other electrode $\left(\mathrm{TiO}_{2}+x \mathrm{Li}^{+}+x \mathrm{e}^{-} \leftrightarrow \mathrm{Li}_{x} \mathrm{TiO}_{2}\right)^{29}$ will move to the forward direction for the same reason, enabling $\mathrm{Li}^{+}$to react with $\mathrm{TiO}_{2}$ so that $\mathrm{Li}_{x} \mathrm{TiO}_{2}$ will be produced at the anode electrode, leaving the positive charges at the $\mathrm{Ti}$ foil as the current collector. During this process, $\mathrm{Li}^{+}$will continuously migrate from the cathode to the anode and the device is charged up a little bit owing to the large volume of the device.

During the progress of charging electrochemical reactions at the two electrodes, extra free electrons will transfer from the cathode to the anode, in order to maintain the charge neutrality and the continuity of the charging reaction. There are generally two ways for the electrons to transfer: either inside the battery system in some manner or through the external circuitry. After comparing the self-charging behavior of the SCPC with and without an outer circuitry (that is the Electrochemical Workstation connected between cathode and anode, to monitor the change of voltage), we suggest that there should probably be some internal mechanisms for the electrons to transfer across the two electrodes (see section $\mathrm{H}$ and Figure S8 


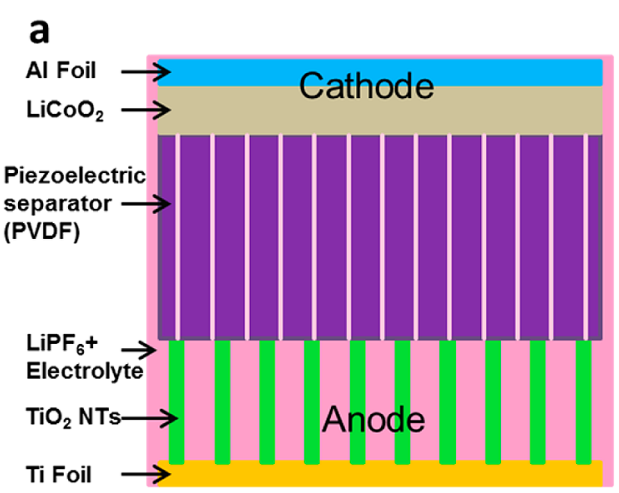

b
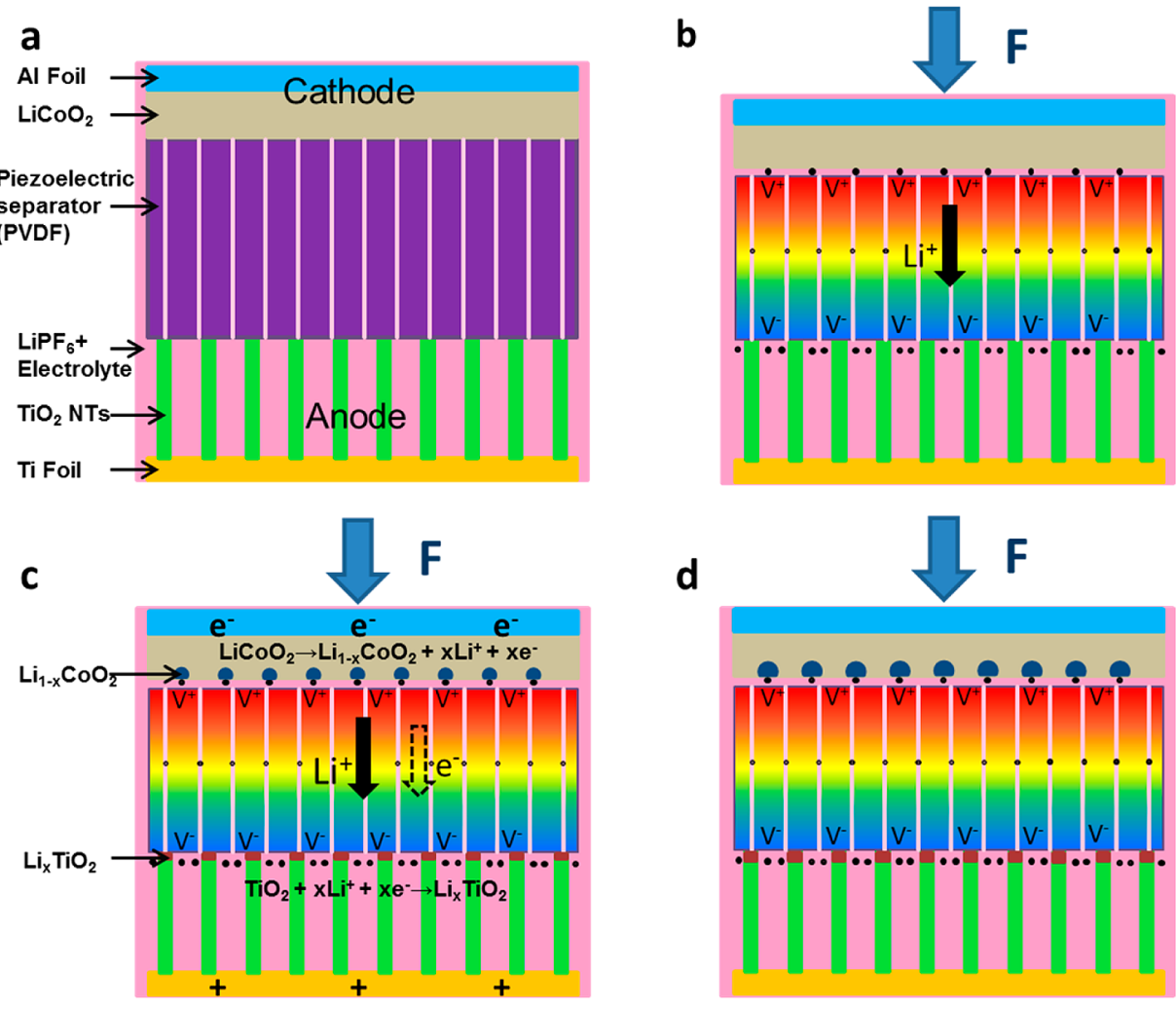

e

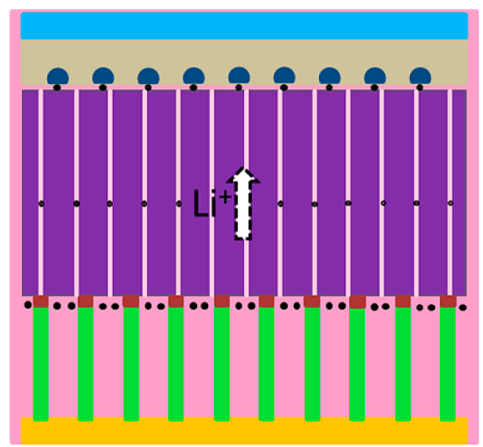

$\mathbf{f}$

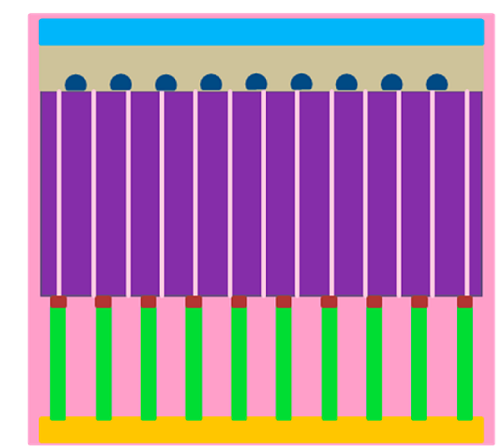

Figure 2. The working mechanism of the self-charging power cell driven by compressive straining. (a) Schematic illustration of the self-charging power cell in discharged state with $\mathrm{LiCoO}_{2}$ as cathode and $\mathrm{TiO}_{2}$ nanotubes as anode. (b) When a compressive stress is applied onto the device, the piezoelectric separator layer (e.g., PVDF film) creates a piezopotential, with the positive polarity at the cathode side and negative piezopotential at the anode. (c) Under the driving of the piezoelectric field, the Li ions from the cathode will migrate through the PVDF film separator in the electrolyte toward the anode, leading to the corresponding charging reactions at the two electrodes. The free electrons at the cathode and positive charges at the anode will dissipate inside the device system. (d) The status where chemical equilibrium of the two electrodes is re-established and the self-charging process ceases. (e) When the applied force is released, the piezoelectric field of the PVDF disappears, which breaks the electrostatic equilibrium, so that a portion of the $\mathrm{Li}$ ions will diffuse back to the cathode. (f) This electrochemical system reaches a new equilibrium, and a cycle of self-charging is completed.

in the Supporting Information), although this exact process is still to be further investigated.

Under the mechanical deformation, the piezopotential continues to drive the migration of $\mathrm{Li}^{+}$ions until a point when the chemical equilibriums of the two electrodes are reestablished and the distribution of the $\mathrm{Li}^{+}$can balance the piezoelectric field in the PVDF film, with no $\mathrm{Li}^{+}$drifting through PVDF (Figure 2d); that is to say, a new equilibrium is achieved and the self-charging process will cease. This is the process of converting mechanical energy directly into chemical energy.

In the second step, when the applied force is released, the piezoelectric field of the PVDF disappears, which breaks the electrostatic equilibrium, so that a portion of the $\mathrm{Li}$ ions diffuse back from the anode to the cathode (Figure 2e) and reach an even distribution of $\mathrm{Li}^{+}$all over the space in the device again (Figure 2f). Then, a cycle of charging is completed through an electrochemical process of oxidizing a small amount of $\mathrm{LiCoO}_{2}$ at the cathode to $\mathrm{Li}_{1-x} \mathrm{CoO}_{2}$ and reducing a bit of $\mathrm{TiO}_{2}$ to $\mathrm{Li}_{x} \mathrm{TiO}_{2}$ at the anode. When the device is mechanically deformed again, the process presented above is repeated, resulting in another cycle of charging by converting mechanical energy directly into chemical energy.

In this self-charging mechanism, the role played by the piezoelectric material (PVDF) is similar to the DC power supply used in the conventional charging process of a $\mathrm{Li}$ 

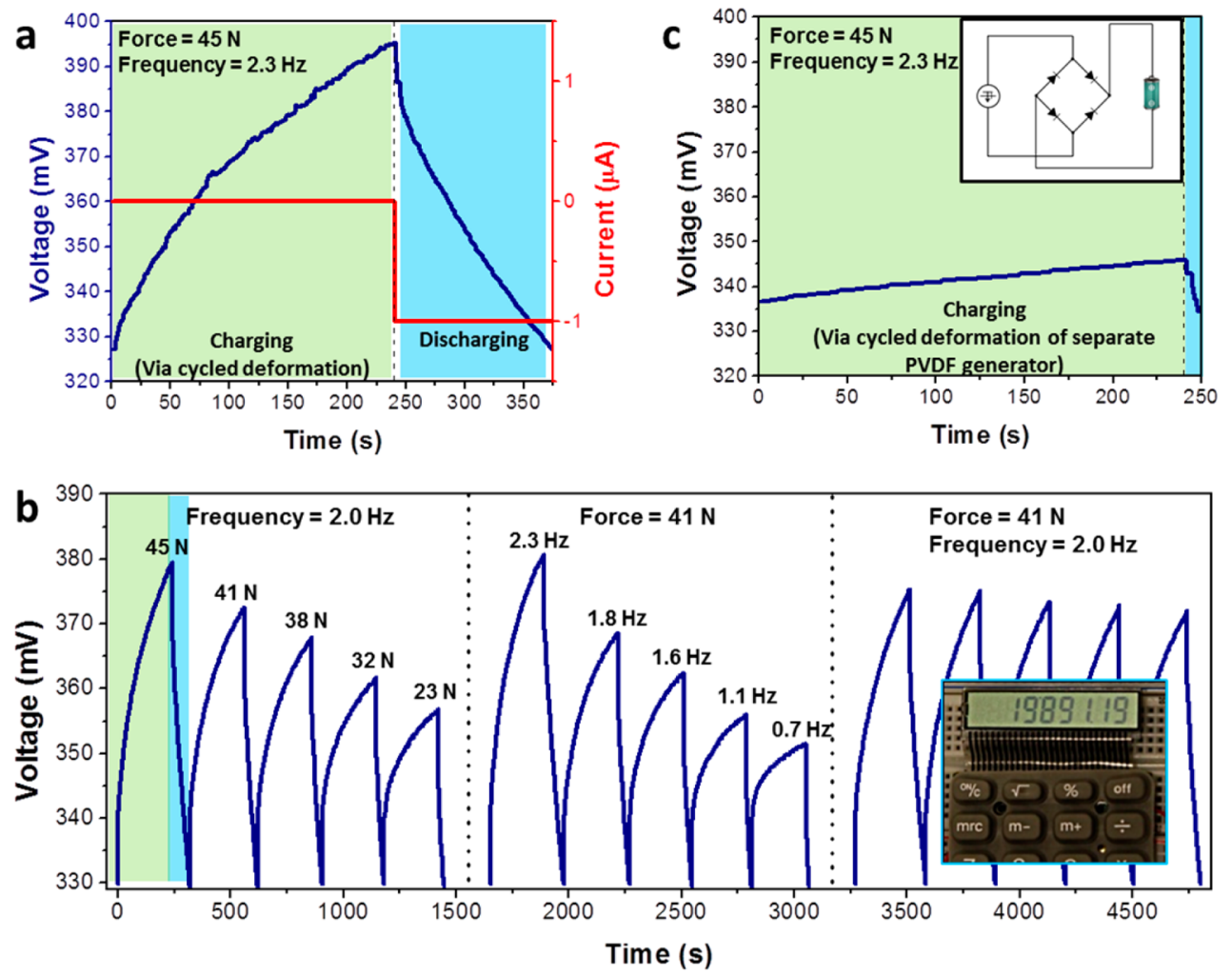

Figure 3. Self-charging process of SCPC under periodic compressive straining and the corresponding discharging process. (a) A typical self-charging process simply by applying cycled mechanical compressive strain to the device (green shadowed region), during which the voltage keeps raising but the current flowing through an external load connected between the cathode and anode remains almost zero, indicating that the charging process is accomplished by the migration of the $\mathrm{Li}$ ions in the internal circuit rather than the flow of electrons in the external load. In the discharge process (blue shadowed region), the stored power is released in the form of electron flow in the external load, as indicated by the measured current and drop of voltage. (b) Self-charging and discharge cycles of SCPC under different forces and frequencies, respectively. Note that the force indicated in the figure was the force applied to the entire device, most of which was consumed at the stainless shell of the cell; only a very small fraction of the force reached the PVDF. The inset shows the operation of a commercial calculator using the SCPCs as the power source. (c) As a comparison of efficiency, the SCPC is separated into two individual units: a PVDF piezoelectric generator and a Li-ion battery by using PE as a separator. This plot shows the voltage across the battery as being charged by the generator for 4 min under the same condition as for part a, followed by a discharge process under the current of $1 \mu \mathrm{A}$. The inset is a schematic circuit of the traditional charging methods with separated generator and storage units connected by a bridge rectifier.

battery. Both of them can be deemed as charge pumps, but the specific mechanisms are different. As for the conventional charging method, the DC power supply will pump the electrons from the positive electrode to the negative electrode through the external circuit and the Li ions will go in the same direction but within the cell, in order to remain a neutral charge balance. Thus, the electrochemical reactions on the two electrodes occur and the battery is charged up. However, for our SCPC proposed here, the piezoelectric material pumps the $\mathrm{Li}^{+}$ions, rather than the electrons, from the positive to the negative electrode, which also accomplishes the charging of the device. This mechanism can also be explained by thermodynamics. According to Nernst's theory, ${ }^{30}$ the relative electrode potentials of the two electrodes have the following relationships with $\mathrm{Li}^{+}$ concentration:

$$
\begin{aligned}
& \varphi_{\mathrm{Li}_{1-x} \mathrm{CoO}_{2} / \mathrm{LiCoO}_{2}}=\varphi_{\mathrm{Li}_{1-x} \mathrm{CoO}_{2} / \mathrm{LiCoO}_{2}}^{\circ}-\frac{R T}{F} \ln \frac{1}{\left[a_{\mathrm{c}}\left(\mathrm{Li}^{+}\right)\right]^{x}} \\
& \varphi_{\mathrm{TiO}_{2} / \mathrm{Li}_{x} \mathrm{TiO}_{2}}=\varphi_{\mathrm{TiO}_{2} / \mathrm{Li}_{x} \mathrm{TiO}_{2}}^{\circ}-\frac{R T}{F} \ln \frac{1}{\left[a_{\mathrm{a}}\left(\mathrm{Li}^{+}\right)\right]^{x}}
\end{aligned}
$$

where $\varphi_{\mathrm{Li}_{1-x} \mathrm{CoO}_{2} / \mathrm{LiCoO}_{2}}$ and $\varphi_{\mathrm{TiO}_{2} / \mathrm{Li}_{x} \mathrm{TiO}_{2}}$ are actual electrode potentials of the cathode and anode, $\varphi_{\mathrm{Li}_{1-x} \mathrm{CoO}_{2} / \mathrm{LiCoO}_{2}}^{\circ}$ and
$\varphi_{\mathrm{TiO}_{2} / \mathrm{Li}_{x} \mathrm{TiO}_{2}}^{\circ}$ are standard electrode potentials of these two electrodes, and $a_{\mathrm{c}}\left(\mathrm{Li}^{+}\right)$and $a_{\mathrm{a}}\left(\mathrm{Li}^{+}\right)$are the activities of $\mathrm{Li}^{+}$ around the cathode and anode, respectively, which can be approximately equated to the concentrations, $R$ is the gas constant, $T$ is the temperature, and $F$ is the Faraday constant. Thus, under the driving of piezoelectric field, because of depletion of $\mathrm{Li}^{+}$concentration near the positive electrode, the electrode potential $\varphi_{\mathrm{Li}_{1-x} \mathrm{CoO}_{2} / \mathrm{LiCoO}_{2}}$ will decrease; likewise, the elevation of $\mathrm{Li}^{+}$concentration will result in an increase of $\varphi_{\mathrm{TiO}_{2} / \mathrm{Li}_{x} \mathrm{TiO}_{2}}$ at the negative electrode. For conventional $\mathrm{Li}$-ion battery, the electrode potential $\varphi_{\mathrm{Li}_{1-x} \mathrm{CoO}_{2} / \mathrm{LiCoO}_{2}}$ is larger than $\varphi_{\mathrm{TiO}_{2} / \mathrm{Li}_{x} \mathrm{TiO}_{2}}$, so that the battery can discharge spontaneously through the reduction of $\mathrm{Li}_{1-x} \mathrm{CoO}_{2}$ and oxidization of $\mathrm{Li}_{x} \mathrm{TiO}_{2}$. However, for the self-charging process, because the change of $\mathrm{Li}^{+}$concentration will possibly make $\varphi_{\mathrm{TiO}_{2} / \mathrm{Li}_{x} \mathrm{TiO}_{2}}$ larger than $\varphi_{\mathrm{Li}_{1-x} \mathrm{CoO}_{2} / \mathrm{LiCoO}_{2}}$, the device is self-charged through the reduction of $\mathrm{TiO}_{2}$ and oxidization of $\mathrm{LiCoO}_{2}$.

By using a mechanical setup that can provide a periodic compressive stress onto the device, we demonstrated the selfcharging process of the power cell. Figure $3 \mathrm{a}$ is a typical selfcharging and discharging cycle. Under the compressive force 

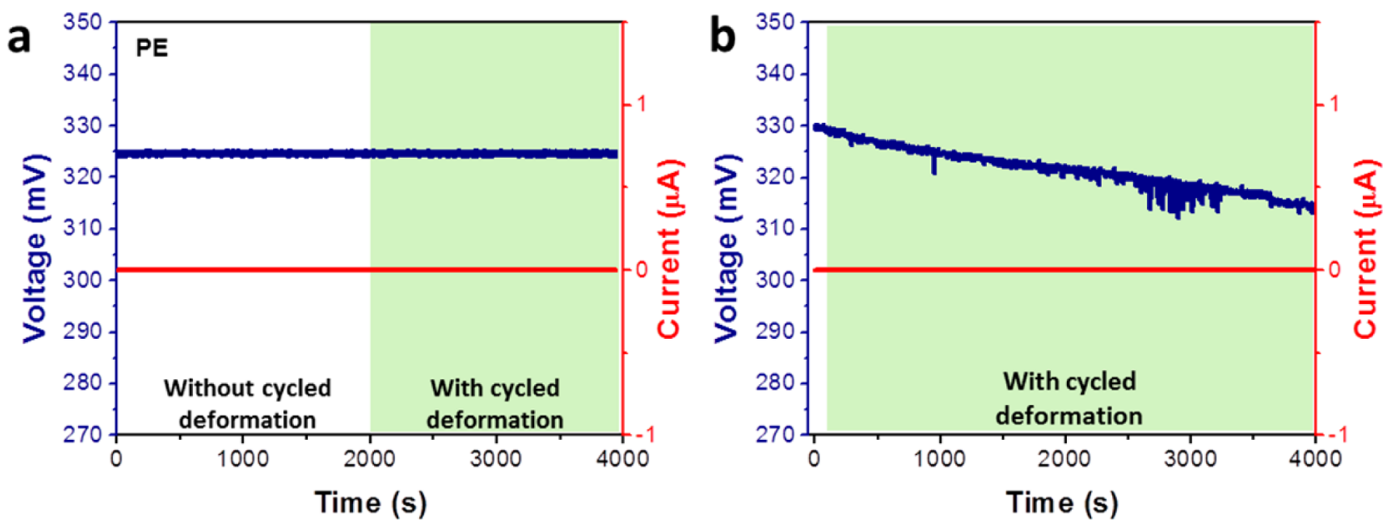

Figure 4. Response of devices of the similar structure as self-charging power cell but with different films as separators. (a) For a conventional Li-ion battery using PE as the separator, no charging effect is observed by applying cycled mechanical deformation, indicating the result presented in Figure $3 \mathrm{a}$ is due to the piezoelectric driven charging process. (b) For a device that has the same structure as a SCPC but with the PVDF film having a piezoelectric field pointing from anode to cathode, there is no charging effect either, just as expected from the mechanism presented in Figure 2.

applied to the SCPC at a frequency of $2.3 \mathrm{~Hz}$, the voltage of the device increased from 327 to $395 \mathrm{mV}$ in $240 \mathrm{~s}$. After the selfcharging process, the device was discharged back to its original voltage of $327 \mathrm{mV}$ under a discharge current of $1 \mu \mathrm{A}$, which lasted for about $130 \mathrm{~s}$. Thus, we proved that the proposed power cell can be charged up under the repeated deformation by directly converting mechanical energy to chemical energy. In this experimental case, the stored electric capacity of the power cell was about $0.036 \mu \mathrm{Ah}$.

From the theory of piezoelectricity, within the elastic deformation regime, the magnitude of the piezopotential is linearly proportional to the magnitude of strain, and thus also to the magnitude of the compressive force (Figure S2, Supporting Information). Figure $3 \mathrm{~b}$ shows that, as we solely increased the mechanical force applied to the device, the selfcharging effect would be enhanced. Thus, an increased piezopotential can give an enhanced charging effect. In addition, the self-charging effect is also affected by the frequency at which the deformation is applied, as shown in Figure $3 \mathrm{~b}$. Under a constant applied force of impact, a higher frequency resulted in an increased charging voltage simply due to higher input power. When the force and the frequency were both kept constant, the rate at which the voltage was increased was relatively stable. The above results are additional proof that the self-charging process is due to the piezoelectric effect. We have demonstrated that a series connection of several SCPCs drove the operation of a commercial calculator for more than $10 \mathrm{~min}$, as shown in the inset of Figure $3 \mathrm{~b}$.

The overall efficiency of our proposed SCPC has two partsthe energy converting efficiency of piezoelectric material and the energy storage efficiency of this mechanical-to-electrochemical process. We compared this with the efficiency of the traditional charging method, which is composed of a separated generator and a storage unit connected though a bridge rectifier (inset of Figure 3c). The generator unit was fabricated by sealing the PVDF film in the same coin cell to create a similar straining condition as SCPC. After being charged for 4 min via cycled deformation of the separate PVDF generator, the voltage of the battery has only increased by $\sim 10 \mathrm{mV}$ (Figure 3c), which is a lot lower than that of SCPC $(65 \mathrm{mV})$ (Figure 3a). Therefore, the single mechanical-to-chemical process for SCPC is much more efficient than the mechanical-to-electric and then electrical-to-chemical double-processes for charging a traditional battery. This is because our current study demonstrated a new approach for directly converting mechanical energy into chemical energy without going through the generation of electricity as an intermediate state, which saves at least the energy wasted on the outer circuitry, including the rectifying component (Figure S11, Supporting Information). This is the innovation of the power cell. Moreover, this self-charging effect also works for SCPC with relatively higher voltage $(\sim 1.5 \mathrm{~V})$, as shown in Figure S9a (Supporting Information). It is clearly shown that the mechanical-to-electrochemical process we proposed here is effective in a wide voltage range, which means that the SCPC could be fully charged on the basis of the mechanism we proposed. From the above experiments, we can see that the SCPC we proposed here could be a powerful device to simultaneously harvest and store mechanical energy, after making several improvements on piezoelectric separators, device structure, fabrication techniques, and development of flexible devices: first, the rigid stainless steel coin cell, which consumed a large portion of mechanical energy, is apparently not an optimal way for the packaging of SCPC. Moreover, the sealing provided by such a coin cell seems to be not good enough under periodic deformation, so that the leakage of a normal coin-cell-type battery will deteriorate when subjected to deformation, which largely affected the performance of SCPC. Second, the straining of piezoelectric material in such device structure is not very effective, which could be solved by designing flexible devices in the future.

As a comparison and control experiment, we measured the response of a conventional Li-ion battery under the same deformation conditions, which had the same structure as the power cell except using PE film as the separator instead of the piezoelectric PVDF film. As shown in Figure 4a, the voltage remained the same at $\sim 325 \mathrm{mV}$ during the $\sim 4000$ cycles of deformation that lasted for $\sim 0.5 \mathrm{~h}$. Thus, the conventional $\mathrm{Li}-$ ion battery cannot be charged up at all by applying cycled mechanical deformation. This is because there is no piezoelectric potential that drives the migration of $\mathrm{Li}$ ions. This comparison experiment rules out the possible contribution from the electrostatic noise or the measurement system to the charging process presented in Figure 3a for SCPC.

Alternatively, if the piezoelectric polarization as presented in Figure 2 is reversed, the piezoelectric field in the PVDF film is pointing from anode to cathode, which will drive the migration of Li ions in the opposite direction. As a result, the reverse electrochemical process will take place under the similar 
mechanism, so that the entire device will be slightly discharged after many cycles of mechanical deformation. The response of the device fabricated using PVDF film with opposite polarization was measured under the same deformation conditions, as shown in Figure $4 \mathrm{~b}$. The voltage slightly decreased from 330 to $315 \mathrm{mV}$ during the $\sim 8000$ cycles of deformation that lasted for $\sim 1.1 \mathrm{~h}$. Rather than self-charging, the self-discharging has been accelerated in such a device simply because the piezopotential drove $\mathrm{Li}$ ions to migrate in the opposite direction of the charging process. This could be more clearly demonstrated on fully charged devices. As shown in Figure S9b (Supporting Information), the piezopotential from periodic deformations can help the device to self-discharge all the full capacity in about 1 day's time, which normally takes several months. This further confirmed the working principle of the SCPC, as proposed in Figure 2, can continuously drive the progress of electrochemical reactions for the energy storage.

In summary, a new mechanical-to-electrochemical process is proposed by integrating piezoelectric material with an electrochemical system, in which an approach for fabricating a selfcharging power cell is demonstrated for converting and simultaneously storing mechanical energy directly as chemical energy, with a significantly higher overall efficiency than the traditional charging method composed of two separated units. By replacing the PE separator as for conventional Li battery with a piezoelectric PVDF film, the piezoelectric potential from the PVDF film created under straining acts as a charge pump to drive $\mathrm{Li}$ ions migrating from the cathode to the anode accompanying charging reactions at electrodes, which can be defined as a piezo-electrochemical process. Using the mechanism demonstrated here, we have hybridized a generator with a battery for the first time as a sustainable power source. It provides an innovative approach for developing new energy technology for driving personal electronics and self-powered systems.

Method. $\mathrm{TiO}_{2}$ nanotube arrays were directly grown on $\mathrm{Ti}$ foils ( $0.05 \mathrm{~mm}$ thick, $99.6 \%$ purity; Alfa Aesar) by electrochemical anodizing in ethylene glycol solution containing 0.3 wt $\% \mathrm{NH}_{4} \mathrm{~F}$ and 2 vol $\% \mathrm{H}_{2} \mathrm{O}$, with $\mathrm{Pt}$ as counter electrode. Prior to growth, all $\mathrm{Ti}$ foils were ultrasonically cleaned in acetone, water, and ethanol consecutively, and then dried in air. A thin layer of PMMA was spin-coated on one side of the foil to protect it from the etching solution. The prepared Ti foil was anodized at $50 \mathrm{~V}$ for $5 \mathrm{~h}$, and then treated by ultrasonication in acetone for a few seconds, leaving hexagon-like footprints on the surface of Ti foil. A second anodization was then performed under the same condition for $2 \mathrm{~h}$ to produce well-aligned $\mathrm{TiO}_{2}$. Finally, the two-step anodized nanotubes were annealed at 450 ${ }^{\circ} \mathrm{C}$ for $2 \mathrm{~h}$ in the air to form an anatase crystalline phase and remove PMMA on the back of Ti foils.

\section{ASSOCIATED CONTENT}

\section{S Supporting Information}

Additional discussions and figures about properties of the polarized PVDF films, crystal structure of as-synthesized $\mathrm{TiO}_{2}$ nanotubes, viability of the self-charging power cell as a battery system, and the mechanism of the self-charging power cell. This material is available free of charge via the Internet at http:// pubs.acs.org.

\section{AUTHOR INFORMATION}

\section{Corresponding Author}

*E-mail: zlwang@gatech.edu.

\section{Author Contributions}

$\S$ These authors contributed equally.

\section{Notes}

The authors declare no competing financial interest.

\section{ACKNOWLEDGMENTS}

This research was supported by DARPA (HR0011-09-C-0142), Airforce, U.S. Department of Energy, Office of Basic Energy Sciences under Award DE-FG02-07ER46394, NSF (CMMI 0403671), and the Knowledge Innovation Program of the Chinese Academy of Sciences (Grant No. KJCX2-YW-M13).

\section{REFERENCES}

(1) Arico, A. S.; Bruce, P.; Scrosati, B.; Tarascon, J. M.; Van Schalkwijk, W. Nanostructured Materials for Advanced Energy Conversion and Storage Devices. Nat. Mater. 2005, 4, 366-377.

(2) Dresselhaus, M. S.; et al. New Directions for Low-dimensional Thermoelectric Materials. Adv. Mater. 2007, 19, 1043-1053.

(3) Wang, Z. L. Towards Self-Powered Nanosystems: From Nanogenerators to Nanopiezotronics. Adv. Funct. Mater. 2008, 18, $3553-3667$.

(4) Oregan, B; Gratzel, M. A Low-Cost, High-Efficiency Solar-Cell Based on Dye-Sensitized Colloidal TiO2 Films. Nature 1991, 353, 737-740.

(5) Law, M.; Greene, L. E.; Johnson, J. C.; Saykally, R.; Yang, P. D. Nanowire Dye-sensitized Solar Cells. Nat. Mater. 2005, 4, 455-459.

(6) Luther, J. M.; et al. Schottky Solar Cells Based on Colloidal Nanocrystal Films. Nano Lett. 2008, 8, 3488-3492.

(7) Tian, B. Z.; et al. Coaxial Silicon Nanowires As Solar Cells and Nanoelectronic Power Sources. Nature 2007, 449, 885-888.

(8) Poudel, B.; et al. High-thermoelectric Performance of Nanostructured Bismuth Antimony Telluride Bulk Alloys. Science 2008, 320, 634-638.

(9) Shao, Z. P.; et al. A Thermally Self-sustained Micro Solid-oxide Fuel-cell Stack with High Power Density. Nature 2005, 435, 795-798.

(10) Wang, Z. L.; Song, J. H. Piezoelectric Nanogenerators Based on Zinc Oxide Nanowire Arrays. Science 2006, 312, 242-246.

(11) Qin, Y.; Wang, X.; Wang, Z. L. Microfibre-nanowire Hybrid Structure for Energy Scavenging. Nature 2008, 451, 809-813.

(12) Yang, R. S.; Qin, Y.; Dai, L. M.; Wang, Z. L. Power Generation with Laterally Packaged Piezoelectric Fine Wires. Nat. Nanotechnol. 2009, 4, 34-39.

(13) Hu, Y. F.; et al. Self-Powered System with Wireless Data Transmission. Nano Lett. 2011, 11, 2572-2577.

(14) Chang, C. E.; Tran, V. H.; Wang, J. B.; Fuh, Y. K.; Lin, L. W. Direct-Write Piezoelectric Polymeric Nanogenerator with High Energy Conversion Efficiency. Nano Lett. 2010, 10, 726-731.

(15) Chan, C. K.; et al. High-performance Lithium Battery Anodes Using Silicon Nanowires. Nat. Nanotechnol. 2008, 3, 31-35.

(16) Idota, Y.; Kubota, T.; Matsufuji, A.; Maekawa, Y.; Miyasaka, T. Tin-based Amorphous Oxide: A High-capacity Lithium-ion-storage Material. Science 1997, 276, 1395-1397.

(17) Tarascon, J. M.; Armand, M. Issues and Challenges Facing Rechargeable Lithium Batteries. Nature 2001, 414, 359-367.

(18) Poizot, P.; Laruelle, S.; Grugeon, S.; Dupont, L.; Tarascon, J. M. Nano-sized Transition-metaloxides As Negative-electrode Materials for Lithium-ion Batteries. Nature 2000, 407, 496-499.

(19) Bruce, P. G.; Scrosati, B.; Tarascon, J. M. Nanomaterials for Rechargeable Lithium Batteries. Angew. Chem., Int. Ed. 2008, 47, 2930-2946.

(20) Armstrong, G.; Armstrong, A. R.; Bruce, P. G.; Reale, P.; Scrosati, B. TiO2(B) Nanowires As An Improved Anode Material for Lithium-ion Batteries Containing LiFePO4 or LiNi0.5Mn1.5O4 Cathodes and A Polymer Electrolyte. Adv. Mater. 2006, 18, 25972600.

(21) Wakihara, M.; Yamamoto, O. Lithium Ion Batteries: Fundamentals and Performance; Kodansha; Wiley-VCH: 1998. 
(22) Xu, S.; et al. Self-powered Nanowire Devices. Nat. Nanotechnol. 2010, 5, 366-373.

(23) Zhang, S. S. A Review on The Separators of Liquid Electrolyte Li-ion Batteries. J. Power Sources 2007, 164, 351-364.

(24) Macak, J. M.; Albu, S. P.; Schmuki, P. Towards Ideal Hexagonal Self-ordering of TiO2 Nanotubes. Phys. Status Solidi RRL 2007, 1, $181-183$.

(25) Salimi, A.; Yousefi, A. A. FTIR Studies of Beta-phase Crystal Formation in Stretched PVDF Films. Polym. Test. 2003, 22, 699-704. (26) Chiang, C. Y.; Shen, Y. J.; Reddy, A. J.; Chu, P. P. Complexation of Poly(vinylidene fluoride): LiPF6 Solid Polymer Electrolyte with Enhanced Ion Conduction in 'Wet' Form. J. Power Sources 2003, 123, 222-229.

(27) Fransson, L.; Eriksson, T.; Edstrom, K.; Gustafsson, T.; Thomas, J. O. Influence of Carbon Black and Binder on Li-ion Batteries. J. Power Sources 2001, 101, 1-9.

(28) Yoshio, M.; Brodd, R. J.; Kozawa, A. Lithium-ion Batteries: Science and Technologies; Springer: 2009.

(29) Zhou, Y. K.; Cao, L.; Zhang, F. B.; He, B. L.; Li, H. L. Lithium Insertion into $\mathrm{TiO} 2$ Nanotube Prepared by The Hydrothermal Process. J. Electrochem. Soc. 2003, 150, A1246-A1249.

(30) Bard, A. J.; Faulkner, L. R. Electrochemical Methods: Fundamentals and Applications, 2nd ed.; John Wiley: 2001. 